Pacific Journal of Mathematics

THE INTRINSIC METRICS ON THE CIRCULAR DOMAINS IN 


\section{THE INTRINSIC METRICS ON THE CIRCULAR DOMAINS IN $\mathbf{C}^{n}$}

\section{MASAAKI SUZUKI}

In this paper we study the intrinsic metrics for the circular domains in $\mathrm{C}^{n}$. We calculate the Kobayashi (pseudo-) metric at its center for pseudoconvex complete circular domain $D$ using the result of Sadullaev. From this we have that such $D$ is hyperbolic iff $D$ is bounded. If a convex complete circular domain is complete hyperbolic, then the Caratheodory and Kobayashi metrics coincide at the center. Using this and the results of Hua we explicitly compute the intrinsic metrics of the classical domains. Furthermore we define the extremal function and extremal disc for intrinsic metrics and compute them in some special cases.

1. Preliminaries. Let $M$ be a (countable, connected) complex manifold, and $T M$ the holomorphic tangent bundle of $M$. Let $\Delta$ denote the unit disc in $\mathbf{C}$ with Poincare metric $d s^{2}=d t \overline{d t} /\left(1-|t|^{2}\right)^{2}$ of Gaussian curvature -4 .

Carathéodory metric (C-metric, in short) $C_{M}(p ; \xi)$ and Kobayashi metric (K-metric, in short) $K_{M}(p ; \xi)$ at $(p ; \xi) \in T M$ are defined by

$$
\begin{gathered}
C_{M}(p ; \xi)=\sup \left\{\left|(d f)_{p} \xi\right| ; f \in \operatorname{Hol}(M, \Delta), f(p)=0\right\}, \\
K_{M}(p ; \xi)=\inf \left\{1 / r: \exists F \in \operatorname{Hol}(\Delta, M), F(0)=p, F^{\prime}(0)=r \xi(r>0)\right\}
\end{gathered}
$$

respectively, where $F^{\prime}(0)=d F_{0}(d / d t)$. (Correctly speaking, these are pseudometrics.) Hereafter we assume $C_{M}>0$ everywhere and $M$ is complete hyperbolic. We work mainly on (bounded) domains in $\mathbf{C}^{n}$. Then $C_{M}$ and $K_{M}$ are continuous Finsler metrics on $M$. For them, we can define the holomorphic curvature as follows (cf. Suzuki [8]). Let $X(p ; \xi)$ denote either $C_{M}(p ; \xi)$ or $K_{M}(p ; \xi)$. We set

$$
H(p ; \xi)=\bigcup_{r>0}\left\{F \in \operatorname{Hol}\left(\Delta_{r}, M\right) ; F^{\prime}(0)=r \xi, F(0)=p\right\} .
$$

We define $\lambda_{F}(t)=X^{2}\left(F(t) ; F^{\prime}(t)\right), t \in \Delta_{r}=\{|t|<r\}$, for each $F$ in $H(p ; \xi)$. Then the holomorphic curvature of $X$ at $(p ; \xi)$ is given by

$$
k_{X}(p ; \xi)=\sup \left\{-\left(2 \lambda_{F}(0)\right)^{-1} \Delta \log \lambda_{F}(0) ; F \in H(p ; \xi)\right\},
$$

where

$$
\Delta \log \lambda_{F}(0)=4 \liminf _{s \rightarrow 0} s^{-2}\left\{\frac{1}{2 \pi} \int_{0}^{2 \pi} \log \lambda_{F}\left(s e^{\imath \theta}\right) d \theta-\log \lambda_{F}(0)\right\} .
$$


Then we have

TheOrem A. ([8]). Assume $C_{M}>0$. Then the holomorphic curvature of $\mathrm{C}-$ metric is $\leq-4$, and that of $\mathrm{K}-$ metric is $\geq-4$. Thus if $C_{M}=K_{M}$, their holomorphic curvatures are -4 .

We may put $C_{M}(p ; 0)=K_{M}(p ; 0)=0$. Fix a point $p$ in $M$. The indicatrices of $\mathrm{C}$ - and $\mathrm{K}$-metrics at $p$ are given by

$$
\begin{aligned}
& I^{C}(M, p)=\left\{\xi \in T_{p} M ; C_{M}(p ; \xi)<1\right\}, \\
& I^{K}(M, p)=\left\{\xi \in T_{p} M ; K_{M}(p ; \xi)<1\right\},
\end{aligned}
$$

respectively. Under our assumptions, they are open sets.

THEOREM B. (cf. [1], [6]). If $M$ is a bounded domain in $\mathbf{C}^{n}$, then $I^{C}(M, p)$ is a bounded convex circular domain with center 0 . If $M$ is a bounded convex complete circular domain in $C^{n}$ with center 0 , then $M=$ $I^{C}(M, 0)$.

2. The extremal function and the extremal disc. Let $M$ be a complex manifold, and fix a $(p ; \xi)$ in $T M(\xi \neq 0)$. Since $\Delta$ is complete hyperbolic, there exists an $f \in \operatorname{Hol}(M, \Delta)$ which attains $C_{M}(p ; \xi)$; $\left|(d f)_{0} \xi\right|=C_{M}(p ; \xi), f(0)=p$. We call such $f$ the extremal function for $(p ; \xi)$.

When $M$ is complete hyperbolic, the family $\operatorname{Hol}(\Delta, M)$ is a normal family and there exists a mapping $F$ in $\operatorname{Hol}(\Delta, M)$ which attains $K_{M}(p ; \xi)$. This mapping $F$ is called an extremal disc for ( $\left.p ; \xi\right)$ (cf. [9]).

Let $f$ and $F$ be an extremal function and an extremal disc for $(p ; \xi)$, respectively. Setting $g=f \circ F$, we have a function $g \in \operatorname{Hol}(\Delta, \Delta)$ with $g(0)=0$. Thus, by the classical Schwarz lemma,

$$
1 \geq\left|g^{\prime}(0)\right|=\left|(d f)_{0}(d F)_{p}\right|=C_{M}(p ; \xi) / K_{M}(p ; \xi) .
$$

Note that if $C_{M}(p ; \xi)=K_{M}(p ; \xi)$ for some $(p ; \xi)$, then $g(t)=e^{\imath \theta} t$, $t \in \Delta, \theta \in R$.

Proposition 1. Let $M$ be complete hyperbolic. If $C_{M}(p ; \xi)=$ $K_{M}(p ; \xi)$ for some $(p ; \xi)$, then an extremal disc $F: \Delta \rightarrow M$ for $(p ; \xi)$ is an isometric immersion with respect to $\mathrm{K}$-metric.

Proof. Since $K_{M}$ is distance decreasing with respect to $F$,

$$
K_{M}\left(F(t) ; d F_{t}(1)\right) \leq K_{\Delta}(t ; 1)=1 /\left(1-|t|^{2}\right) \text { for } t \in \Delta .
$$


Taking an extremal function $f$ for $(p ; \xi)$, we have

$$
K_{M}\left(F(t) ; d F_{t}(1)\right) \geq K_{\Delta}\left(f \circ F(t) ; d(f \circ F)_{t}(1)\right)=K_{\Delta}(t ; 1)
$$

because $f \circ F(t)=e^{i \theta} t$. Hence we have $K_{M}\left(F(t) ; d F_{t}(1)\right)=K_{\Delta}(t ; 1)$.

An extremal disc $F(\Delta)$ is an analytic disc in $M$ to which the restriction of the K-metric of $M$ coincides with the Poincaré metric. We shall exhibit in the next section the extremal functions and discs for the ball and polydisc.

3. The complete circular domains in $\mathbf{C}^{n}$. In this section we assume $M$ is a complete circular domain $D$ in $\mathbf{C}^{n}$ with center 0 (i.e. $t D \subset D$ for any $t \in \mathbf{C},|t| \leq 1)$. Let $l_{\xi}$ be a complex line in direction $\xi$ passing through 0 . We denote the radius of the disc $1_{\xi} \cap D$ by $R(\xi)$. Note that $R(t \xi)=$ $R(\xi)$ for any $t \in \mathbf{C}-\{0\}$. We define the function $r: D \rightarrow[0,+\infty]$ by $r(z)=\|z\| / R(z)$ if $z \neq 0$ and $r(0)=0$. Then $r$ is upper semicontinuous and $D$ is represented by $D=\left\{z \in \mathbf{C}^{n} ; r(z)<1\right\}$. (\| $\|$ is the Euclidean norm of $\mathbf{C}^{n}$.)

We shall calculate the $\mathrm{K}-\left(\right.$ pseudo) metric at $(0 ; \xi) \in T D=D \times \mathbf{C}^{n}$ $(\xi \neq 0)$ for the pseudoconvex complete circular domain $D$. We recall the Schwarz lemma by Sadullaev [7].

Lemma C. (1) Let $F=\left(F_{1}, \ldots, F_{n}\right)$ be a holomorphic mapping of the unit disc $\Delta$ into a pseudoconvex complete circular domain $D$ and $F(0)=0$. Then $\|F(t)\| \leq R(F(t))|t|$ for $t \in \Delta$.

(2) Under the above conditions, the linear part of $F$ maps $\Delta$ into $D$.

We take a holomorphic mapping $F$ in $\operatorname{Hol}(\Delta, D)$ such that $F(0)=0$ and $d F_{0}(d / d t)=F^{\prime}(0)=r \xi$ for some $r>0$. By Lemma $\mathrm{C}(2)$, the linear part $F^{\prime}(0) t$ of $F$ maps $\Delta$ into $D$, and from (1) we have $\left\|F^{\prime}(0) t\right\| \leq R\left(F^{\prime}(0) t\right)$ $|t|$ for $t \in \Delta$. Since $R\left(F^{\prime}(0) t\right)=R(\xi)$, we have $1 / r \geq\|\xi\| R(\xi)^{-1}$. On the other hand, $K_{D}(0 ; \xi)$ is the infimum of such $1 / r$. Thus $K_{D}(0 ; \xi)=$ $\|\xi\| R(\xi)^{-1}$.

PROPOSITION 2. If D is a pseudoconvex complete circular domain in $\mathbf{C}^{n}$, $D$ is hyperbolic if and only if $D$ is bounded.

Proof. Let $D$ be unbounded. Then we can take a sequence $\left\{z_{k}\right\}$ in $D$ such that $\left\|z_{k}\right\| \rightarrow+\infty, z_{k} \neq 0$. Setting $\xi_{k}=z_{k} /\left\|z_{k}\right\|$ we have a sequence $\left\{\xi_{k}\right\}$ with $\left\|\xi_{k}\right\|=1$, and we may assume $\left\{\xi_{k}\right\}$ accumulates to a point $\xi$ 
$(\|\xi\|=1)$. By completeness of $D, R\left(\xi_{k}\right) \geq\left\|z_{k}\right\|$, hence $K_{D}\left(0 ; \xi_{k}\right)=$ $R\left(\xi_{k}\right)^{-1} \rightarrow 0$ as $k \rightarrow+\infty$. This means $D$ is not hyperbolic at $(0 ; \xi)$. We proved that if $D$ is hyperbolic then $D$ is bounded. The converse is trivial.

REMARK. Kodama [5] has proved the above without assuming pseudoconvexity.

THEOREM 1. Let D be a complete circular domain in $\mathbf{C}^{n}$ with center 0 . If $D$ is complete hyperbolic, then

$$
\begin{gathered}
K_{D}(0 ; \xi)=\|\xi\| R(\xi)^{-1} \quad(\xi \neq 0), \\
I^{C}(D, 0) \supset I^{K}(D, 0)=D .
\end{gathered}
$$

Furthermore, if $D$ is convex, then

$$
C_{D}(0 ; \xi)=K_{D}(0 ; \xi)=\|\xi\| R(\xi)^{-1} \text { for all } \xi \in \mathbf{C}^{n}-\{0\} .
$$

Proof. When $D$ is complete hyperbolic, $D$ is taut and pseudoconvex (cf. [4]), and by Proposition 2, D is bounded. The indicatrix of the $\mathrm{K}$-metric at center 0 is

$$
I^{K}(D, 0)=\left\{\xi \in \mathbf{C}^{n} ; K_{D}(0 ; \xi)<1\right\}=\left\{\xi \in \mathbf{C}^{n} ; r(\xi)<1\right\}=D .
$$

Since $C_{D}(0 ; \xi) \leq K_{D}(0 ; \xi)$ in general, we have $I^{C}(D, 0) \supset I^{K}(D, 0)=D$. As we have noted in $\S 1$, if $D$ is bounded convex, then $I^{C}(D, 0)=D$; hence $I^{C}(D, 0)=I^{K}(D, 0)=D$. In this case we have $C_{D}(0 ; \xi)=K_{D}(0 ; \xi)$ for all $\xi \neq 0$. In fact if $C_{D}\left(0 ; \xi_{0}\right)<K_{D}\left(0 ; \xi_{0}\right)$ for some $\xi_{0} \neq 0$, we can take a real number $s$ such that $C_{D}\left(0 ; \xi_{0}\right)<s<K_{D}\left(0 ; \xi_{0}\right)$. Then $C_{D}\left(0 ; s^{-1} \xi_{0}\right)<$ $1<K_{D}\left(0 ; s^{-1} \xi_{0}\right)$. Thus $s^{-1} \xi_{0} \in I^{C}(D, 0)$, but $s^{-1} \xi_{0} \notin I^{K}(D, 0)$, a contradiction.

If a bounded circular domain is homogeneous, then it is a symmetric domain (Vigué [10]). The bounded symmetric domains $D$ are convex complete circular domains. Thus by Theorem $1, C_{D}(0 ; \xi)=K_{D}(0 ; \xi)$. From homogeneity it follows that $C_{D}(z ; \xi)=K_{D}(z ; \xi)$ for all $(z ; \xi)$ in $T D$. These results are contained in Kobayashi $[3,4]$.

Corollary. Let $D$ be a bounded symmetric domain in $\mathbf{C}^{n}$. Then the $\mathrm{C}-$ and $\mathrm{K}$-metrics of $\mathrm{D}$ coincide on the tangent bundle and their holomorphic curvatures are -4 . 
EXAmple 1. We take the unit ball $D=\left\{z \in \mathbf{C}^{n} ;\|z\|<1\right\}$. Then $R(\xi)=1, K_{D}(0 ; \xi)=\|\xi\|$. Let $F \in \operatorname{Hol}(\Delta, D)$ be an extremal disc for $(0, \xi)$. Then $F(0)=0, \quad F^{\prime}(0)=\xi\|\xi\|^{-1}$. For $t \in \Delta$, we set $h(t)=$ $\left(F(t), F^{\prime}(0)\right)$, where $($,$) is the Hermitian inner product of \mathbf{C}^{n}$. Then $h(0)=0$,

$$
|h(t)| \leq\|F(t)\| \cdot\left\|F^{\prime}(0)\right\| \leq|t|<1, \quad \text { and } \quad\left|h^{\prime}(0)\right|=\left\|F^{\prime}(0)\right\|^{2}=1 .
$$

Hence by Schwarz's lemma, $h(t) \equiv t$ on $\Delta$. We obtain a unique extremal disc for $(0 ; \xi), F(t)=\left(\xi_{1}\|\xi\|^{-1} t, \ldots, \xi_{n}\|\xi\|^{-1} t\right)$. On the other hand, the unit ball is symmetric. Thus $K_{D}(0 ; \xi)=C_{D}(0 ; \xi)$. Let $f(z)$ be an extremal function for $(0 ; \xi)$. Then $f \circ F(t)=e^{i \theta} t, t \in \Delta$. If $\xi_{1}, \ldots, \xi_{n} \neq 0, f(z)=$ $e^{i \theta} \Sigma \xi_{j} z_{j}\|\xi\|^{-1}$. When some $\xi_{j}=0$, an extremal function is not unique.

EXample 2. Now we consider the polydisc $D=\left\{z \in \mathbf{C}^{n} ;\left|z_{j}\right|<1\right.$, $j=1, \ldots, n\}$. In this case $R(\xi)=\|\xi\|\left(\max \left|\xi_{j}\right|\right)^{-1}$. Hence $K_{D}(0 ; \xi)=$ $\max \left|\xi_{j}\right|$. If the above maximum is achieved for only one index $j$, the extremal function for $(0 ; \xi)$ is

$$
f(z)=\left(\bar{\xi}_{j} /\left|\xi_{j}\right|\right) z_{j}=e^{i \theta} z_{j} .
$$

For example, for $j=1$, the extremal disc $F(t)=\left(F_{1}(t), \ldots, F_{n}(t)\right)$ for $(0 ; \xi)$ is given by

$$
\begin{gathered}
F_{1}(t)=e^{i \theta} t, \\
F_{j}(t)=t\left(\operatorname{tg}_{j}(t)+a_{j}\right) /\left(1+\bar{a}_{j} \operatorname{tg}(t)\right), \quad j=2, \ldots, n,
\end{gathered}
$$

where $g_{j}(t) \in \operatorname{Hol}(\Delta, \Delta)$ and $a_{j}=\xi_{j} / \xi_{1}$ (cf. Stanton [9]).

4. The bounded symmetric domains. We consider the intrinsic metrics of the bounded symmetric domains of classical type. Let $M(m, n)$ denote the set of all $(m, n)$-matrices and write $M(n, n)=M(n)$.

$$
\begin{aligned}
R_{\mathrm{I}}(m, n) & =\left\{Z \in M(m, n) ; E_{n}-Z^{*} Z>0\right\}, \\
R_{\mathrm{II}}(n) & =\left\{Z \in M(n) ; Z^{\prime}=Z, E_{n}-Z^{*} Z>0\right\}, \\
R_{\mathrm{III}}(n) & =\left\{Z \in M(n) ; Z^{\prime}=-Z, E_{n}-Z^{*} Z>0\right\}, \\
R_{\mathrm{IV}}(n) & =\left\{Z \in M(n, 1) ; 1-2 Z^{*} Z+\left|Z^{\prime} Z\right|^{2}>0,\left|Z^{\prime} Z\right|<1\right\},
\end{aligned}
$$

where $E_{n}$ is the unit matrix and $Z^{\prime}$ is the transpose of $Z$ and $Z^{*}=\bar{Z}^{\prime}$. These are complete circular domains with center 0 , and convex. Let $R$ or $R_{j}$ denote one of these domains. The group $G$ of biholomorphic transformations of $R$ acts on $R$ transitively, thus we may consider $C_{R}(0 ; \xi)$ or $K_{R}(0 ; \xi)$ only. Let $G_{0}$ be the isotropy subgroup at 0 . 
Lemma D (Hua [2]) (1). For $\xi \in M(m, n)$, there is a $g \in G_{0}$ such that

$$
g \xi=\left(\begin{array}{ccc}
\lambda_{1} & & 0 \\
& \ddots & \\
0 & & \lambda_{n}
\end{array}\right), \quad \lambda_{1} \geq \cdots \geq \lambda_{n} \geq 0
$$

where $\lambda_{1}^{2}, \ldots, \lambda_{n}^{2}$ are eigenvalues of $\xi^{*} \xi$.

(2) For $\xi \in M(n)$ with $\xi^{\prime}=\xi$, there is a $g \in G_{0}$ such that

$$
g \xi=\operatorname{diagonal}\left[\lambda_{1}, \ldots, \lambda_{n}\right], \quad \lambda_{1} \geq \cdots \geq \lambda_{n} \geq 0,
$$

where $\lambda_{1}^{2}, \ldots, \lambda_{n}^{2}$ are eigenvalues of $\xi^{*} \xi$.

(3) For $\xi \in M(n)$ with $\xi^{\prime}=-\xi$, there is a $g \in G_{0}$ such that

$$
g \xi=\text { diagonal }\left[\left(\begin{array}{cc}
0 & \lambda_{1} \\
-\lambda_{1} & 0
\end{array}\right), \ldots,\left(\begin{array}{cc}
0 & \lambda_{k} \\
-\lambda_{k} & 0
\end{array}\right), 0\right]
$$

where $k=[n / 2], \lambda_{1}^{2} \geq \cdots \geq \lambda_{k}^{2} \geq 0$ are eigenvalues of $\xi^{*} \xi$, and the last term is 0 if $n$ is odd.

(4) For $\xi \in M(n, 1)$, there exists a $g \in G_{0}$ such that

$$
g \xi=\left(\lambda_{1}, i \lambda_{2}, 0, \ldots, 0\right),
$$

where $\lambda_{1}^{2}, \lambda_{2}^{2}$ are eigenvalues of the $(2,2)$ matrix

$$
\left(\begin{array}{c}
\operatorname{Re} \xi^{\prime} \\
\operatorname{Im} \xi^{\prime}
\end{array}\right)(\operatorname{Re} \xi, \operatorname{Im} \xi)
$$

Theorem 2. Let $C_{j}$ and $K_{j}$ denote the C-metric and $\mathrm{K}$-metric of $R_{J}$ respectively. Then, for $j=\mathrm{I}$, II, III,

$$
\begin{aligned}
C_{j}(0 ; \xi) & =K_{j}(0 ; \xi) \\
& =\max \left\{\text { positive square roots of the eigenvalues of } \xi^{*} \xi\right\}
\end{aligned}
$$

and

$$
C_{\mathrm{IV}}(0 ; \xi)=K_{\mathrm{IV}}(0 ; \xi)=\left(\|\xi\|^{2}+\left(\|\xi\|^{4}-\left|\xi^{\prime} \xi\right|^{2}\right)^{1 / 2}\right)^{1 / 2}
$$


Proof. Since the proofs are almost the same for $j=$ I, II, III, we prove only the case $R_{\mathrm{I}}$. By Lemma $\mathrm{D}(1)$, we may assume

$$
\xi=\left(\begin{array}{ccc}
\lambda_{1} & & \\
& \ddots & 0 \\
0 & & \lambda_{n}
\end{array}\right), \quad \lambda_{1} \geq \cdots \geq \lambda_{n} \geq 0
$$

and $\lambda_{1}>0$. We show $K_{\mathrm{I}}(0 ; \xi)=\lambda_{1}$. Defining the mapping $F \in$ $\operatorname{Hol}\left(\Delta, R_{\mathrm{I}}\right)$ by

$$
\Delta \ni t \rightarrow\left(\begin{array}{cccc}
t & & & \\
& t \lambda_{2} / \lambda_{1} & & 0 \\
& & \ddots & \\
0 & & t \lambda_{n} / \lambda_{1}
\end{array}\right) \in R_{\mathrm{I}}
$$

we have $d F_{0}\left(\lambda_{1} d / d t\right)=\xi, F(0)=0$. Since $K_{\mathrm{I}}$ is metric decreasing with respect to $F, K_{\mathrm{I}}(0 ; \xi) \leq K_{\Delta}\left(0 ; \lambda_{1}\right)=\lambda_{1}$. Similarly, for the mapping $f$ : $R_{\mathrm{I}} \ni Z \rightarrow z_{11} \in \Delta$, where $Z=\left(z_{i j}\right), d f_{0}(\xi)=\lambda_{1}$ and $f(0)=0$. Hence we have $K_{\mathrm{I}}(0 ; \xi) \geq K\left(0 ; \lambda_{1}\right)=\lambda_{1}$. Thus we obtain $K_{\mathrm{I}}(0 ; \xi)=\lambda_{1}$.

To calculate $K_{\mathrm{IV}}(0 ; \xi)$, we use Theorem 1 . We substitute $z=\xi t\|\xi\|^{-1}$, $|t|=r$ into the inequalities

$$
1-2\|z\|^{2}+\left|\sum z_{j}^{2}\right|^{2}>0, \quad\left|\sum z_{J}^{2}\right|<1 .
$$

Then the radius $R(\xi)$ is the supremum of the $r$ satisfying these inequalities. Easy calculations show

$$
R(\xi)=\|\xi\|\left(\|\xi\|^{2}-\left(\|\xi\|^{4}-\left|\xi^{\prime} \xi\right|^{2}\right)^{1 / 2}\right)^{1 / 2},
$$

and noting that $K_{\mathrm{IV}}(0 ; \xi)=\|\xi\| R(\xi)^{-1}$, we arrive at the conclusion.

Remarks. (1) For any ( $z ; \xi)$ in $T R$, taking a $g \in G$ with $g(z)=0$, we have $K_{j}(z ; \xi)=K_{j}\left(0 ; d g_{z}(\xi)\right)$.

(2) We obtain the same result for $K_{\mathrm{IV}}(0 ; \xi)$ from the calculation using the fact $\left\{\left(z_{1}+i z_{2}, z_{1}-i z_{2}\right), z=\left(z_{1}, \ldots, z_{n}\right) \in R_{\mathrm{IV}}(n)\right\}$ is the geodesic polydisc in $R_{\mathrm{IV}}(n)$ (cf. [4]), and Lemma $\mathrm{D}(4)$.

The author is grateful to A. Kodama and K. Azukawa for very helpful conversations. 


\section{REFERENCES}

[1] C. Carathéodory, Über die geometrie der analytischen Abbildungen, die durch analytische Functionen von zwei Vänderlichen vermittelt werden, Abh. Math. Semin. Hamburg, Bd 6 (1928), 96-145.

[2] L. K. Hua, On the theory of automorphic functions of a matrix variable I. Amer. J. Math., 66 (1944), 470-488.

[3] S. Kobayashi, Hyperbolic manifolds and holomorphic mappings, Pure and Appl. Math., 2.

[4] Intrinsic distances, measures and geometric function theory, Bull. Amer. Math. Soc., 82 (3) (1976), 357-416.

[5] A. Kodama, On boundedness of circular domains, Proc. Japan Acad., (to appear).

[6] H. J. Reiffen, Die differentialgeometrischen Eigenshaften der invarianten Distanz funktion von Carathéodory, Schr. Math. Inst. Univ. Münster. No. 26.

[7] A. Sadullaev, Schwarz lemma for circular domains and its applications, Math. Note, 27 (1980), 120-125.

[8] M. Suzuki, The holomorphic curvature of intrinsic metrics, Math. Rep. Toyama Univ., 4 (1981), 107-114.

[9] C. M. Stanton, A characterization of polydisc, Math. Ann., 253 (1980), 129-135.

[10] J. P. Vigué, Le groupe des automorphismes analytiques $d$ un domaine borne $d$ un espace de Banach complexe application aux domaines bornes symmetriques, Ann. Scient. Ec. Norm. Sup., 9 (1976), 203-282.

Received August 31, 1982.

TOYAMA UNIVERSITY

GOFUKU, TOYAMA, JAPAN 


\section{PACIFIC JOURNAL OF MATHEMATICS \\ EDITORS}

DONALD BABBITT (Managing Editor)

University of California.

Los Angeles, CA 90024

Hugo Rossi

University of Utah

Salt Lake City, UT 84112

C. C. Moore and Arthur Ogus

University of California

Berkeley, CA 94720
J. DugundjI

Department of Mathematics

University of Southern California

Los Angeles, CA 90089-1113

R. FinN and H. Samelson

Stanford University

Stanford, CA 94305

\section{ASSOCIATE EDITORS}
R. ARENS
E. F. BECKENBACH
B. H. NeumanN
F. WOLF
K. YoshidA (1906-1982)

\section{SUPPORTING INSTITUTIONS}

UNIVERSITY OF ARIZONA

UNIVERSITY OF BRITISH COLUMBIA

CALIFORNIA INSTITUTE OF TECHNOLOGY

UNIVERSITY OF CALIFORNIA

MONTANA STATE UNIVERSITY

UNIVERSITY OF NEVADA. RENO

NEW MEXICO STATE UNIVERSITY

OREGON STATE UNIVERSITY
UNIVERSITY OF OREGON

UNIVERSITY OF SOUTHERN CALIFORNIA

STANFORD UNIVERSITY

UNIVERSITY OF HAWAII

UNIVERSITY OF TOKYO

UNIVERSITY OF UTAH

WASHINGTON STATE UNIVERSITY

UNIVERSITY OF WASHINGTON 


\section{Pacific Journal of Mathematics}

Vol. 112, No. $1 \quad$ January, 1984

Richard Blaine Barrar and Henry Loeb, Characterizing the divided difference weights for extended complete Tchebycheff systems $\ldots \ldots \ldots \ldots 1$

Harold Bennett and David John Lutzer, Generalized ordered spaces with

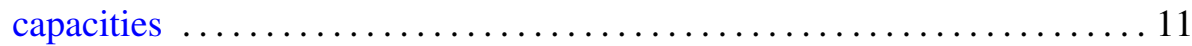

Geoffrey J. Butler and Lynn Harry Erbe, Comparison theorems for second-order operator-valued linear differential equations

Bohumil Cenkl and Richard D. Porter, de Rham theorem with cubical

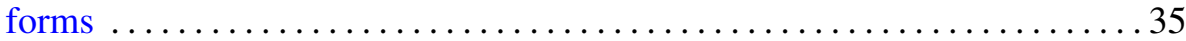

Zvonko Cerin, Characterizing global properties in inverse limits . ....... 49

Thomas Eugene Duchamp and Morris Kalka, Holomorphic foliations and deformations of the Hopf foliation .........................69 69

John Paul Hempel, Homology of coverings $\ldots \ldots \ldots \ldots \ldots \ldots \ldots \ldots \ldots$

Gerald Norman Hile and R. Z. Yeh, Inequalities for eigenvalues of the

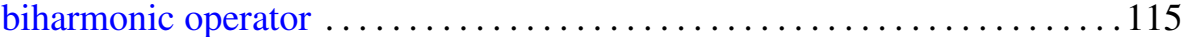

Kenneth Irwin Joy, A description of the topology on the dual space of a

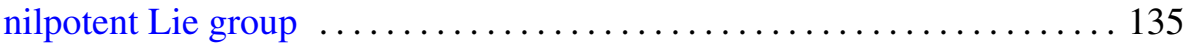

Alex Kumjian, On localizations and simple $C^{*}$-algebras $\ldots \ldots \ldots \ldots \ldots 141$

Bernardus de Pagter, The space of extended orthomorphisms in a Riesz

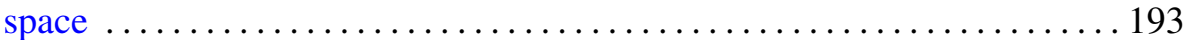

Stephen C. Persek, Iterated averaging for periodic systems with hidden

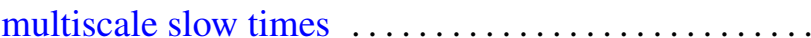

David Francis Rearick, Divisibility of arithmetic functions . . . . . . . . . 237

Masaaki Suzuki, The intrinsic metrics on the circular domains in $\mathbf{C}^{n}$ 